Article

\title{
Effects of Grafting Azacrown Ether on Thermal and Swelling Properties of Chitosan Films
}

\author{
Julius Toeri ${ }^{1,2, *}$ (D) and Marie-Pierre Laborie ${ }^{1,2}$ \\ 1 Chair of Forest Biomaterials, University of Freiburg, Werthmanstr. 6, 79085 Freiburg, Germany; \\ marie-pierre.laborie@biomat.uni-freiburg.de \\ 2 Freiburg Materials Research Center, University of Freiburg, Stefan-Meier-Straße 21, 79104 Freiburg, Germany \\ * Correspondence: ratumo.toeri@biomat.uni-freiburg.de or ratumojt@gmail.com; Tel.: +49-761-203-3764
}

Received: 20 September 2017; Accepted: 15 November 2017; Published: 17 November 2017

\begin{abstract}
The thermal and swelling properties of a series of azacrown ether-crosslinked chitosans prepared with varying molar amounts of $N, N$-diallyl-7,13-diaza-1,7,10,16-tetraoxa-dibenzo-18-crown-6 (molar equivalents ranging from $0,0.125,0.167,0.25$ and 0.5 ) films were studied with Thermographimetric analysis (TGA), Differential Scanning Calorimetry (DSC), Dynamic Mechanical analysis (DMA) techniques and swelling kinetics. Introducing the azacrown (DAC) as crosslinker into the chitosan matrices $(\mathrm{Ch})$ altered the thermal and swelling properties of the chitosan/crown ether films (Ch-DAC) systematically with respect to molar ratios. At lower DAC content, a depression of $T_{g}$ revealed a dominating internal plasticization effect of DAC on chitosan, while higher DAC molar ratios systematically increased the $T_{g}$ of the network. The films high swelling capacity (as high as $1200 \%$ ) was reached within three hours in aqueous acidic media and decreased systematically with increasing DAC content. The swelling behavior was highly dependent on $\mathrm{pH}$ and followed second order kinetics. Understanding the thermal and swelling properties of this series of azacrown ether-crosslinked chitosans sets the stage to further shed light on their impact for heavy metal adsorption in water remediation applications.
\end{abstract}

Keywords: azacrown ether; chitosan crosslinking; glass transition temperature; swelling behavior; chitosan films

\section{Introduction}

Chitosan (Ch), which is easily derived from chitin by $N$-deacetylation, has been tested for application in the removal of toxic heavy metals from contaminated wastewater sources. However, $\mathrm{Ch}$ is soluble in acid media and must therefore be crosslinked for these application. The selective chelating properties exhibited by crown ethers towards metal ions have led to their incorporation into chitosan polymer matrices to improve capacity for metal ion adsorption [1-3], enhance selective pollutant removal [4,5], and thus wastewater remediation [6,7]. While many authors have chemically modified crosslinked chitosan with crown ethers [5,6,8-17], and shown the advantage of combining crown ethers with chitosan $[5,8,9,18,19]$, only a few have used the crown ether as the crosslinker for chitosan $[7,18,20-24]$ and fewer have used the nitrogen containing azacrown ethers [25]. Using the azacrown ether as the crosslinker has the advantage that the consumption of the $-\mathrm{NH}_{2}$ chelating group of the chitosan might be compensated by the azacrown ether chelating property. Furthermore, nitrogen containing azacrown ethers are particularly interesting due to their stronger complexation properties for heavy-metal ions [1,26-28] than the all-oxygen crowns, which strongly complex alkali and alkaline earth metal [26,28]. For example, Ding et al. [25] used $N, N^{\prime}$-diallyl dibenzo-18-crown- 6 crown to crosslink chitosan and revealed that these materials performed better in metal ion adsorption than neat $\mathrm{Ch}$ owing to the presence of the crown ethers $[15,16,22,23]$. Structural 
elucidation of the products suggested that these crown ethers crosslink chitosan, although no report of gel content or network formation was presented in this work and others $[10,11,15,16,20,23]$.

Following on the work of Ding et al. [25], we reported in a previous study on the preparation, chemical and microstructural characterization of azacrown ether crosslinked chitosan films [29]. Stoichiometric control allowed designing a series of modified Ch with $N, N$-diallyl-7,13-diaza1,7,10,16-tetraoxa-dibenzo-18-crown-6 ether (DAC) in controlled molar ratios from Ch:DAC = 1:0, 1:0.125, 1:0.167, 1:0.25, and 1:0.5. Furthermore, high gel content and spectroscopic evidence confirmed that the azacrown ether did also play the role of the crosslinker. How the extent of Ch:DAC ratio in this series of azacrown ether crosslinked chitosan impacts physical, thermal and swelling properties remains unknown. In particular, the swelling behavior and thermal transitions, both aspects being of considerable significance for crosslinked hydrogels, has not been studied systematically for crown ether-modified Ch materials [30,31].

In this publication, we investigate the impact of Ch:DAC molar ratio on the physical, thermal and swelling properties in a series of DAC-crosslinked chitosan films. We thus show that porosity, thermal transitions and swelling behavior of Ch:DAC composites all change systematically up to a threshold DAC content, at which point the trend is reversed. We discuss these trends in light of the parallel mechanisms and competition between branching/grafting and crosslinking by DAC on chitosan.

\section{Materials and Methods}

\subsection{Materials}

This starting chitosan polymer $(\mathrm{Ch})$ from shrimp shell chitin was supplied by Sigma-Aldrich (Germany) and previously characterized by Toeri et al. [29]. It displayed number-average (Mn) and molecular weights $(\mathrm{Mw})$ of $8.202 \times 10^{4}( \pm 0.57 \%) \mathrm{g} / \mathrm{mol}$ and $1.810 \times 10^{5}( \pm 0.30 \%) \mathrm{g} / \mathrm{mol}$, respectively. It had a polydispersity index (PDI) of 2.21. The degree of acetylation and crystallinity index were determined as $23 \%$ and $75.7 \%$ respectively by Toeri et al. [29]. The azacrown ether crosslinking agent, N,N-diallyl-7,16-diaza-1,4,10,13-tetraoxa-dibenzo-18-crown-6 (DAC) was synthesized and characterized by Toeri and Laborie [32]. Other reagents were of analytical grade and used as received.

\subsection{Preparation of Azacrown Ether/Chitosan Hydrogel Films}

The procedure of preparing the chitosan-azacrown ether films (Ch-DAC) was previously reported [29]. Briefly, purified $\mathrm{Ch}(1.0 \mathrm{~g})$ was dissolved in deionized water containing $2 \%(v / v)$ acetic acid and heated to $40^{\circ} \mathrm{C} .5 \mathrm{~mL}$ of iron (III) chloride hexahydrate solution was added and the mixture was stirred for 15 min under nitrogen flow. A required amount of $N, N$-diallyl-dibenzo-18-crown- 6 dissolved in ethanol was slowly dropped into the mixture so that at the end a $2 \mathrm{wt}$. \% solid content was achieved. The resulting mixture was then refluxed at $40{ }^{\circ} \mathrm{C}$ under continuous stirring for $24 \mathrm{~h}$, followed by addition of $5 \mathrm{~mL} \mathrm{H} \mathrm{H}_{2} \mathrm{O}_{2}$ solution. After the reaction was completed, $10 \mathrm{~mL}$ aliquots of the reaction slurry were transferred onto cylindrical glass Petri dishes $(50 \mathrm{~mm} \mathrm{~W} \times 10 \mathrm{~mm} \mathrm{H}$, Pyrex, Corning, Inc. New York, NY, USA) and dried in air for two days followed by oven drying at $60{ }^{\circ} \mathrm{C}$ for $24 \mathrm{~h}$. The formed films were peeled off the glass plates and neutralized by immersion in a $\mathrm{NaHCO}_{3}$ solution. Then, they were washed to $\mathrm{pH} 7$ to give pale white products. The wet films were extracted with ethanol in a Soxhlet extractor for $4 \mathrm{~h}$ to eliminate any unreacted crown ether followed by extraction with aqueous ( $2 \%$ ) acetic acid for $22 \mathrm{~h}$ to eliminated uncrosslinked chitosan. Five formulations designated as Ch-DAC (0), Ch-DAC (0.125), Ch-DAC (0.167), Ch-DAC (0.25), and Ch-DAC (0.5) based on increasing DAC mole ratio to one mole of $\mathrm{Ch}$ deacetylated repeat units were manufactured. Their chemical structure, gel content, and microstructural characterization were determined as previously reported [29]. 


\subsection{Specific Surface Area, Pore Size, and Density of the Films}

Film porosity was characterized by the BET method $\left(\mathrm{N}_{2}\right.$ gas adsorption/desorption method at $77 \mathrm{~K}$ using a Thermo Scientific Sorptomatic 1990 instrument, Freiburg, Germany). Prior to measurements, the samples were outgassed for $5 \mathrm{~h}$ under vacuum at $110{ }^{\circ} \mathrm{C}$. The specific surface area $\left(S_{D R}\right)$ and the micropore volume $\left(V_{\text {micro }}\right)$ of the studied samples was estimated using the Dubinin Radushkevich (DR) method in the linear regression from $\log ^{2}\left(P_{0} / P\right)=0$ to 40.96 [33]. The total pore volume $\left(V_{t}\right)$ was also estimated from the amount of adsorbed $\mathrm{N}_{2}$ at $P / P_{0}=0.95$. All calculations were executed using the Advanced Data Processing 3.0 software. The densities $(\rho)$ of the films were calculated as per Equation (1) using the sample volume $\left(V_{p}\right)$ recorded on a Quanta Chrome micropycnometer instrument (Germany) at $25^{\circ} \mathrm{C}$ with helium gas using Equation (2):

$$
\rho=\frac{m}{V_{p}} \mathrm{~g} / \mathrm{cm}^{3}
$$

where $m$ is the mass of the sample and;

$$
V_{p}=V_{C}-V_{R}\left[\left(P_{1} / P_{2}\right)-1\right]
$$

where $V_{c}$ is the volume of the empty sample chamber, $V_{R}$ is the volume of the reference volume, $P_{1}$ is the first pressure (i.e., in the sample chamber only) and $P_{2}$ is the second pressure after expansion of the gas into the combined volumes of sample chamber and reference chamber.

\subsection{Thermal Analysis}

\subsubsection{Thermogravimetric Analysis (TGA)}

Thermogravimetric analysis was carried out on a Pyris 1 Thermogravimetric analyzer, Perkin Elmer, Waltham, MA, USA. The moisture content was evaluated by the decrease of sample weight at the end of an isotherm by heating the samples to $80^{\circ} \mathrm{C}$ under nitrogen gas $(20 \mathrm{~mL} / \mathrm{min})$ using a heating rate of $10^{\circ} \mathrm{C} / \mathrm{min}$; at this temperature, an isotherm was kept for $60 \mathrm{~min}$. To study the films degradation with temperature, samples were heated under $\mathrm{N}_{2}\left(20 \mathrm{~mL} / \mathrm{min}\right.$ flow rate) from 30 to $900{ }^{\circ} \mathrm{C}$, using a temperature ramp of $10^{\circ} \mathrm{C} / \mathrm{min}$.

\subsubsection{Differential Scanning Calorimetry (DSC)}

The films were oven dried at $80{ }^{\circ} \mathrm{C}$ for at least $24 \mathrm{~h}$ to a moisture content of $1 \%$ or less as determined by TGA. About $6-8 \mathrm{mg}$ dried samples was placed in aluminum pans and heated under nitrogen flow in a differential scanning calorimetry (DSC 850 apparatus, Perkin Elmer, Waltham, MA, USA) in two cycles; from -40 to $180^{\circ} \mathrm{C}$ (first cycle) and -40 to 250 second cycle at $10^{\circ} \mathrm{C} / \mathrm{min}$. The second heating thermograms were used to report the thermal transition temperatures.

\subsubsection{Dynamic Mechanical Analysis (DMA)}

Oven dried rectangular films $\left(20 \times 3 \times 0.1 \mathrm{~mm}^{3}\right)$ with up to $1 \%$ moisture content were tested under tension in a PerkinElmer Instrument DMA 800 within the linear viscoelastic range (displacement amplitude $0.003 \mathrm{~mm}$ ). A temperature sweep was performed at $2{ }^{\circ} \mathrm{C} / \mathrm{min}$ from 20 to $180^{\circ} \mathrm{C}$, under a frequency of $1 \mathrm{~Hz}$.

\subsection{Swelling Behavior}

Swelling kinetics were acquired on films approximately $2 \times 2 \mathrm{~cm}$ by monitoring mass increase upon immersion in water solutions ranging from $\mathrm{pH}=2$ to 10 at $22-23{ }^{\circ} \mathrm{C}$. The $\mathrm{pH}$ of the water was adjusted using $1.0 \mathrm{M} \mathrm{HCl}$ and $\mathrm{NaOH}$ solutions and monitored with a $\mathrm{pH}$ electrode. Sampling occurred every $30 \mathrm{~min}$ for the first $6 \mathrm{~h}$ and thereafter $24 \mathrm{~h}$ and $48 \mathrm{~h}$. The samples were carefully blotted to 
remove excess surface water and immediately weighed. After $48 \mathrm{~h}$, the hydrogel samples were oven dried to constant weight. The water uptake was then calculated as:

$$
\begin{gathered}
\qquad \text { Swelling ratio }(Q)=\frac{W_{t}-W_{0}}{W_{0}} \\
\text { Equilibrium Water Content, } \operatorname{EWC}(\%)=\left[\frac{W_{h}-W_{d}}{W_{h}}\right] \times 100,
\end{gathered}
$$

where $W_{t}$ is the weight of the swelling hydrogel at a time $t, W_{0}$ is the initial weight, $W_{h}$ is the weight of fully swollen hydrogel at equilibrium and $W_{d}$ is the weight of dried hydrogel after swelling.

\section{Results}

\subsection{Films Characterization: Film Porosity, Specific Surface Area, and Density}

Neat chitosan exhibits low porosity $\left(0.7634 \mathrm{~m}^{2} \mathrm{~g}^{-1}, 0.00051 \mathrm{~cm}^{3} \mathrm{~g}^{-1}\right.$, and $0.00027 \mathrm{~cm}^{3} \mathrm{~g}^{-1}$ for $S_{D R}$, $V_{t}$, and $V_{\text {micro }}$, respectively). It increases rapidly by four- to five-fold upon reaction with crown ether up to a Ch:DAC of 0.167 (Figure 1). Past this threshold, porosity decreases again with DAC content albeit at a lower rate. This trend reveals that competing mechanisms govern the porosity of the Ch-DAC films as composition changes. An initial porosity increase of chitosan materials with increasing DAC content might be explained by the dominating grafting effect of a few DAC, while further DAC increase led to a densification, possibly due to dominating crosslinking or packing additional DAC within the crosslinked chitosan network.

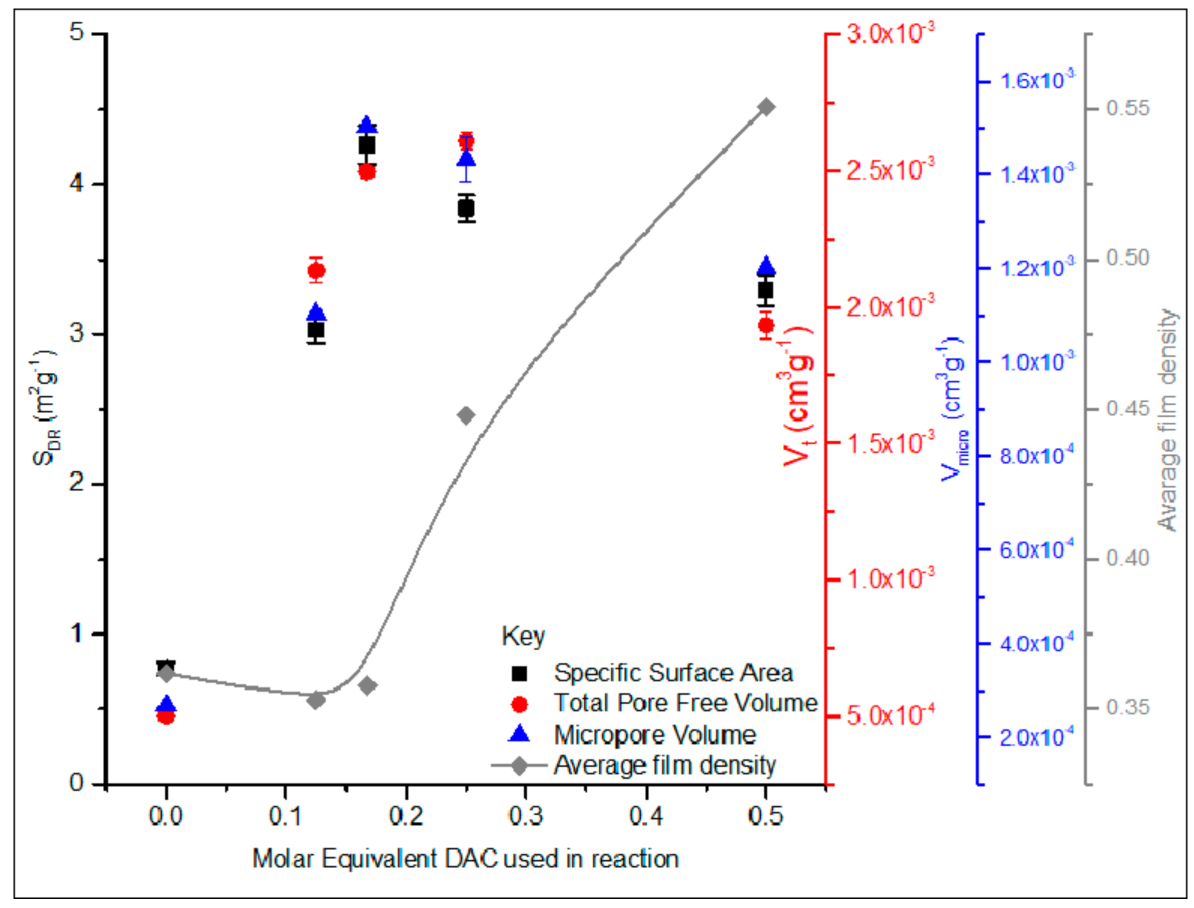

Figure 1. Relationship between molar equivalent $N, N$-diallyl-7,16-diaza-1,4,10,13-tetraoxa-dibenzo-18crown-6 (DAC) against specific surface area, total pore volume, micropore volume, and average film density. 


\subsection{Thermal Properties of the Synthesized Ch-DAC Films}

\subsubsection{TGA Analysis}

Figure $2 \mathrm{a}, \mathrm{b}$ reveals a thermal degradation behavior of Ch-DAC intermediate to that of neat DAC and neat $\mathrm{Ch}$. Noticeably, neat chitosan and Ch-DAC films with DAC content up to 0.167 , display one degradation temperature $\left(\mathrm{T}_{\mathrm{D} 1}\right)$ between $315^{\circ} \mathrm{C}$ and $318^{\circ} \mathrm{C}$ (Figure $\left.2 \mathrm{~b}\right)$. At a higher DAC ratio $(>0.167)$, a second degradation temperature appears $\left(\mathrm{T}_{\mathrm{D} 2}\right.$ at $337^{\circ} \mathrm{C}$ and $\left.347^{\circ} \mathrm{C}\right)$, which is close to the degradation temperature of neat DAC at $\sim 340{ }^{\circ} \mathrm{C}$; [32]. This suggests that it is possible to experimentally distinguish the $\mathrm{Ch}$ degradation from the DAC degradation at sufficiently high DAC contents.

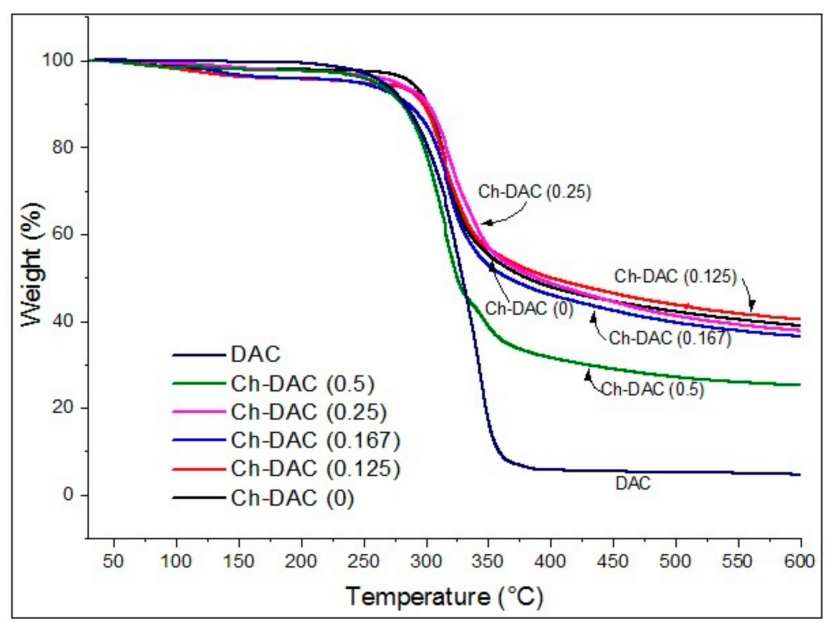

(a)

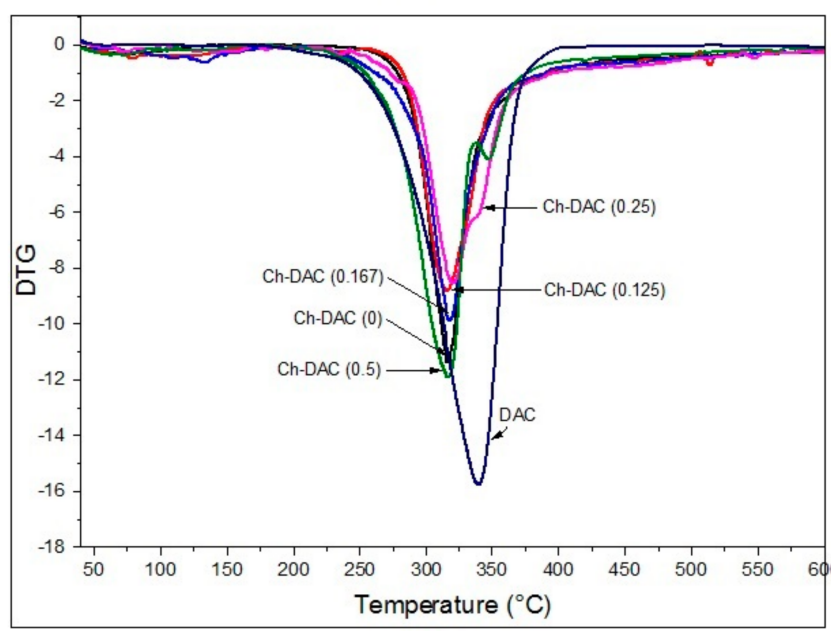

(b)

Figure 2. (a) Thermographimetric analysis thermograms of the chitosan/crown ether films (Ch-DAC) films and (b) second degradation temperature (DTG) curves of the films.

\subsubsection{DSC Analysis}

A single glass transition temperature $\left(T_{g}\right)$ is observed for all Ch-DAC (Figure 3), revealing that the films form single phase composites over the whole range of compositions. By comparison to chitosan, reacting chitosan with low DAC content ratios up to $[\mathrm{DAC}]=0.125$, initially depresses the $T_{g}$ from $136^{\circ} \mathrm{C}$ to $108^{\circ} \mathrm{C}$ (see also Table S1). Recall that prior to analyses, all these have been thoroughly extracted with ethanol and aqueous acetic acid to remove any soluble fractions. The remaining gel fraction has (at low DAC) a lower $T_{g}$ than the chitosan itself. This is well known behavior when grafting a short or bulky 
group/chain on a linear polymer. We thus conclude that at this low DAC content, the gel (thus of course crosslinked) has a significant fraction of grafts/branches that play the usual role of internal plasticizer. This effect obviously predominates the $T_{g}$ increase (as to be expected) from crosslinking (Scheme 1).

With DAC contents above $0.125 \mathrm{~mol} / \mathrm{mol}$, the $T_{g}$ of the films increases again systematically, revealing that crosslinking density increases. Also note that the melting transition characteristic of neat DAC at $124^{\circ} \mathrm{C}$ [32] is not detected in any composite formulations, including in high DAC content formulations, for which TGA revealed a characteristic degradation of bulk DAC phases. Despite the detection of bulk DAC domains by TGA, these DAC domains apparently do not develop crystallinity as was confirmed with the WAXS studies [29].

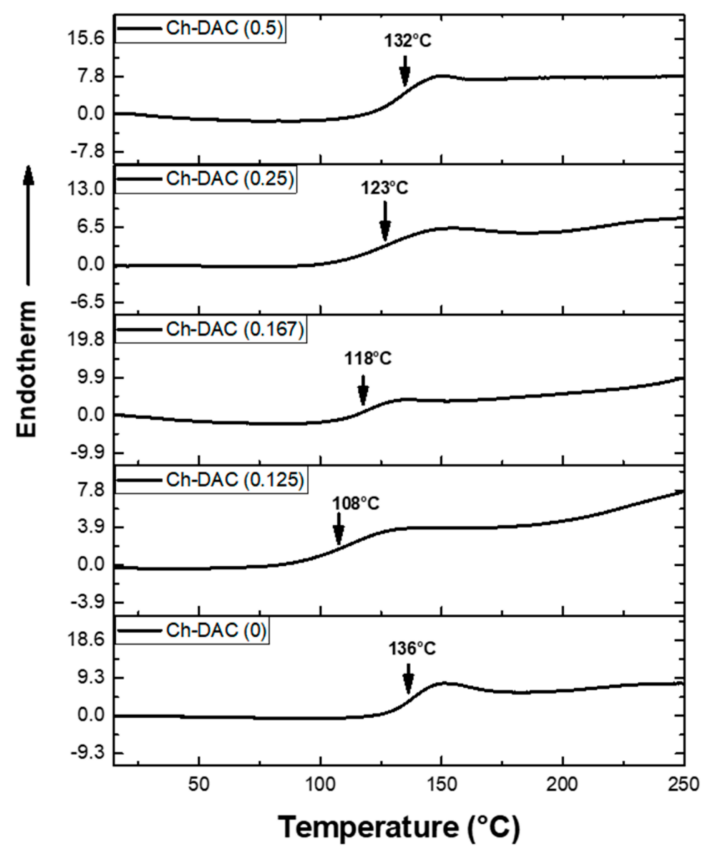

Figure 3. Differential scanning calorimetry thermograms of Ch-DAC films showing a decrease and then an increase in the film glass transition temperature as a function of DAC content.

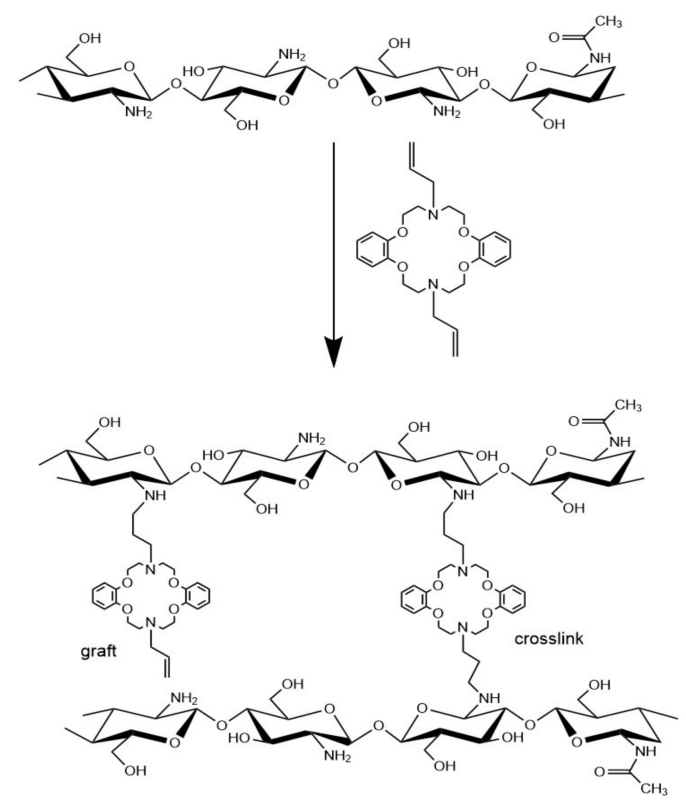

Scheme 1. Ch-DAC reaction showing possible DAC grafting and crosslinking [29]. 


\subsubsection{DMA Analysis}

The DMA thermograms reveal a major relaxation associated with drop in $\mathrm{E}^{\prime}$ at $150{ }^{\circ} \mathrm{C}$ for neat chitosan, which is generally ascribed to the alpha relaxation (Figure 4). All Ch-DAC films also exhibit this relaxation between 135 and $150{ }^{\circ} \mathrm{C}$. At the lowest DAC content, the relaxation of chitosan is lowered to ca. $137^{\circ} \mathrm{C}$. With increasing DAC content, this temperature gradually rises back to $150{ }^{\circ} \mathrm{C}$. This behavior is in line with that observed with DSC. The initial drop in the glass transition of modified chitosan with low DAC content confirms the initial plasticizing effect of DAC on chitosan, while the gradual rise in $T_{g}$ and glassy modulus with increasing DAC loading supports the formation of crosslinked chitosan networks and stiffening of the composite. Also note a secondary relaxation in neat chitosan around $\sim 86^{\circ} \mathrm{C}$, which is generally attributed to the water-induced $\beta$ relaxation $[34,35]$ and is still noticeable in the composite samples, without major changes.

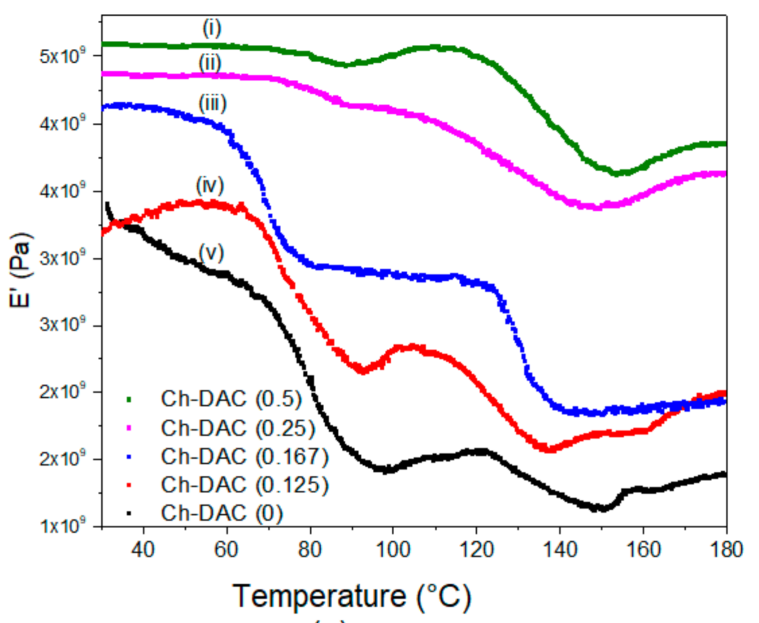

(a)

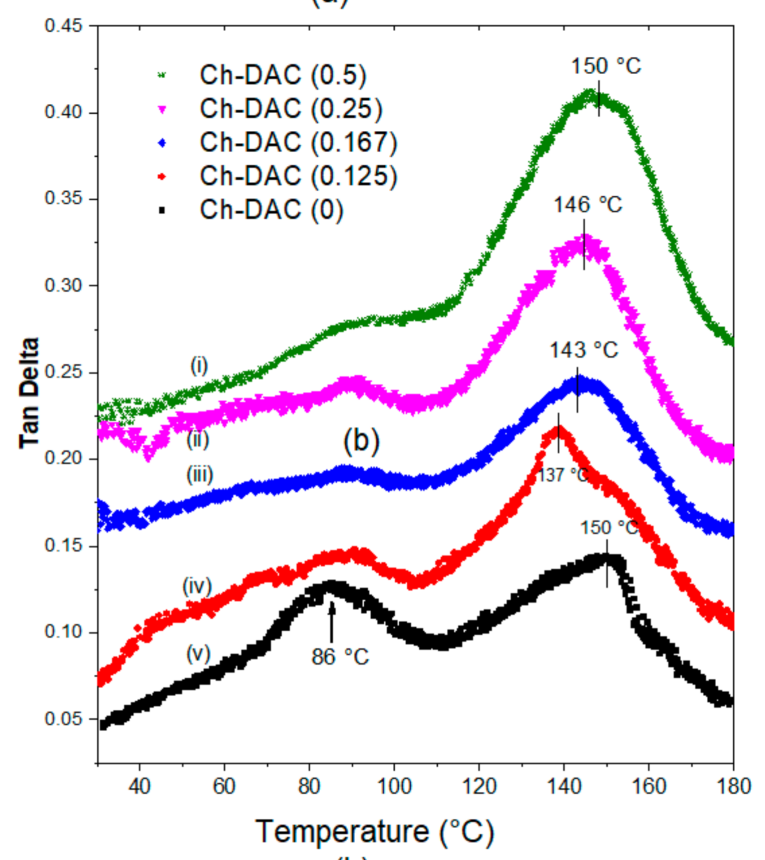

(b)

Figure 4. (a) Dynamic mechanical thermograms of the films showing the effect of increasing the crown ether ratio on the $T_{g}$ of the films (b) tensile storage modulus ( $\left.E^{\prime}\right)$ of the films: from (i) to (v) is decreasing DAC content from $0.5,0.25,0.167,0.125$, and 0 , respectively. 
Figure 5 summarizes all main transitions observed in the series of Ch-DAC. It highlights that the main transitions adopt a different behavior below and above a threshold DAC content around 0.125 molar ratio, and thus the occurrence of competing mechanisms below and above this threshold.

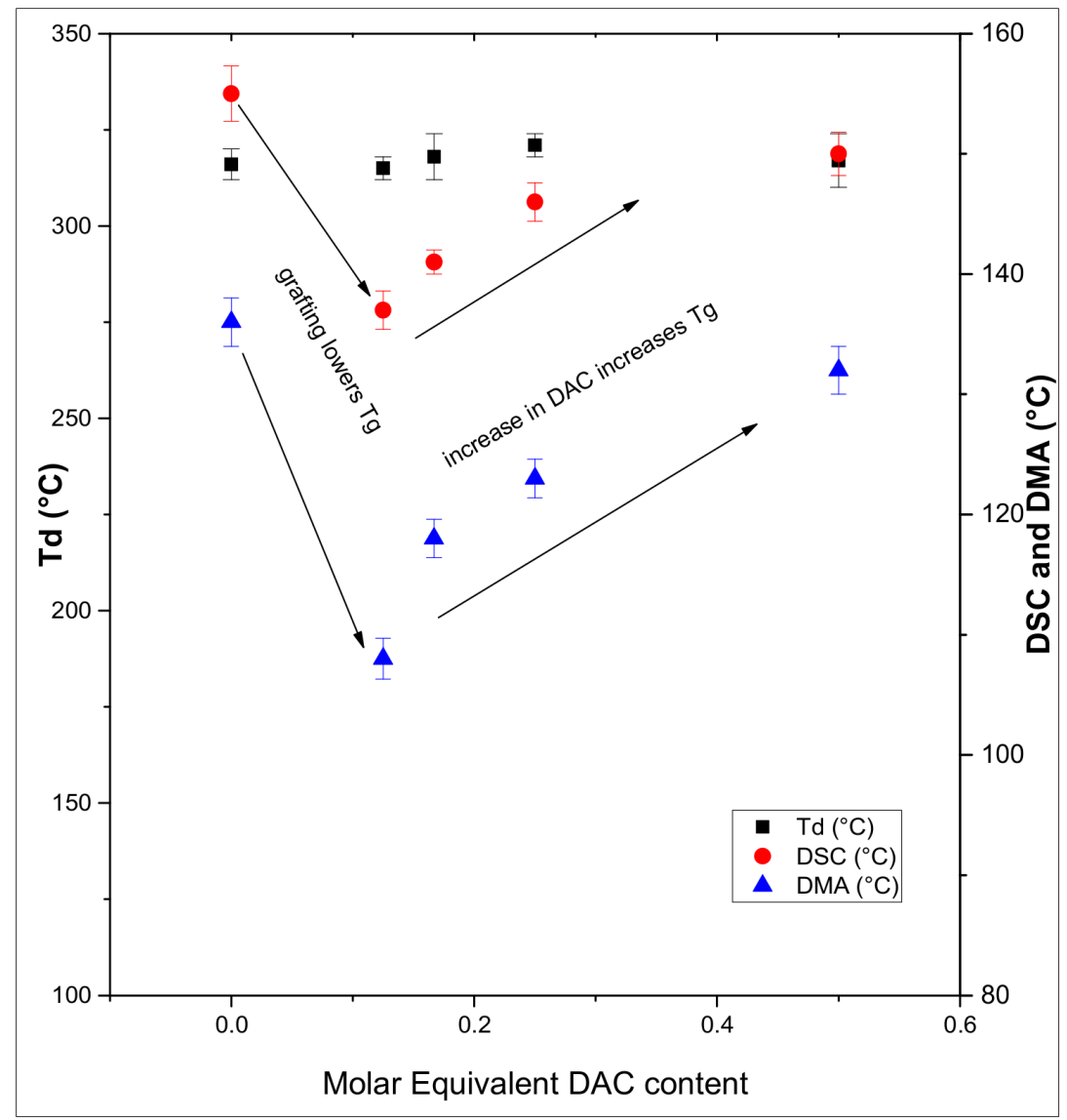

Figure 5. Changes in the thermal behavior (thermal degradation, TD1, and glass transition temperature, $T_{g}$ ) of neat chitosan and Ch-DAC film derivatives as a function of crown ether reacting ratio.

\subsection{Swelling Behavior}

\subsubsection{Water Adsorption Behavior of Films in Various pH Conditions}

A key characteristic of networks are their swelling properties, which is governed by crosslinking density and in the case of chitosan by external factors such as $\mathrm{pH}$. Swelling ratio and equilibrium water content for the films with increasing DAC content in various $\mathrm{pH}$ water solutions $(2,4,7,8$, and 10) are shown in Figure 6 (see also Table S2, Figures S1 and S2 respectively). No swelling was performed for neat chitosan films in water solutions of $\mathrm{pH} 2$ and 4 because they would dissolve easily. It is observed that almost all films attained an EWC (equilibrium water content) of $\sim 60 \%$ and above. This shows that the films had a high swelling ability. With 0.125 molar DAC content the swelling capacity increases at all $\mathrm{pHs}$, and most dramatically at the neutral and acidic $\mathrm{pH}$. For example, at a $\mathrm{pH}$ of 7 , grafting $0.125 \mathrm{~mol}$ of DAC onto chitosan raises the swelling capacity of neat chitosan from $200 \%$ to $600 \%$. Further increasing [DAC] molar content up to 0.5 moles per mole chitosan leads to gradual decline in water adsorption capacity (Figure 6). Also, water adsorption properties strongly depended on $\mathrm{pH}$ and grafting ratios. In acidic $\mathrm{pH}$ solutions, ranging from 2 to 7 , film matrices generally produced the highest degree of swelling in the order of $\mathrm{pH}=2>4>7$ viz-a-viz each graft formulation, and consistently decreased from a maximum of $\sim 1140 \%$ for the lowest $\mathrm{pH}$ of 2 to a value of $126 \%$ for $\mathrm{pH} 7$ as the graft ratio increased. At basic $\mathrm{pH}$, the swelling capacity is little affected by the molar ratios. This strong 
$\mathrm{pH}$ dependency of swelling can be explained by the fact that below the $\mathrm{pK}_{\mathrm{a}}$ value of 6.5 amine groups in the chitosan molecules are ionized to ammonium ion $\left(-\mathrm{NH}_{3}^{+}\right)$[36]. These cationic charges in fiber structure act as repulsive forces between polymer chains, allowing more swelling [37]. Raising the $\mathrm{pH}$ above 7 reduces the number of $-\mathrm{NH}_{3}^{+}$groups and increases the number of $\mathrm{NH}_{2}$ groups. Swelling is thus reduced as the positive charge repulsions are removed. This is clear in the films with DAC content of $0.167,0.25$, and 0.5 mole ratios for $\mathrm{pH} 7$ and above. From a high of $\sim 600 \%$ for the former $([\mathrm{DAC}]=0.167, \mathrm{pH} 7)$, swelling decreases to about $200 \%$ or lower $[\mathrm{DAC}]=0.25$ and above, $\mathrm{pH} 8$ and 10). Interestingly, it can also be noted that there is a slight increase in swelling going from $\mathrm{pH} 8$ to 10 . When the solution $\mathrm{pH}$ increases, the deprotonated amine groups and electron rich DAC central cavity create more negative charge density sites leading to electrostatic repulsion which tends to expand the network [38].

Swelling of DAC derivatives mostly above $600 \%$ without collapsing into solution confirms the chemical stability of these films in solution. This stability might be needed during metal ion adsorption applications from aqueous media with $\mathrm{pH}$ range of 2-10.

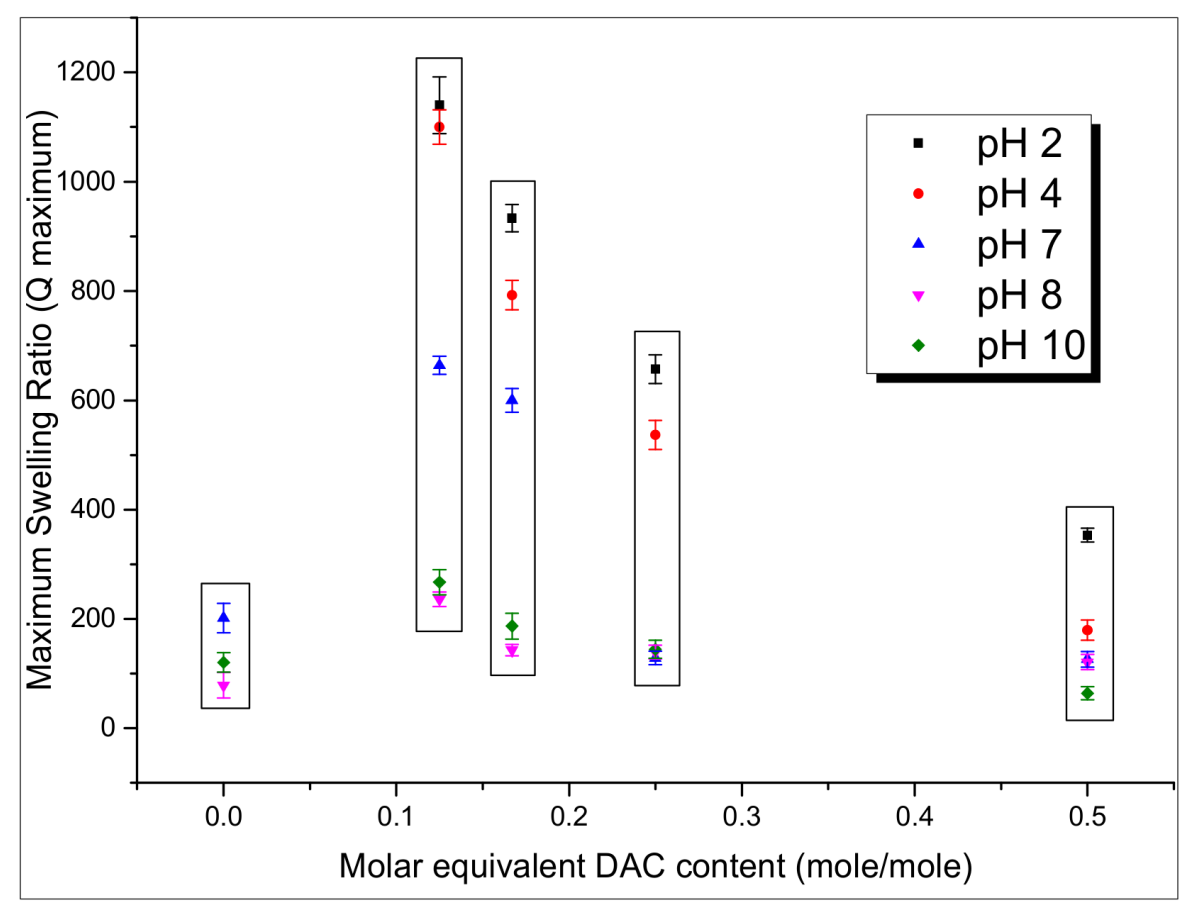

Figure 6. Maximum swelling capacity for Ch-DAC films reacted with $0,0.125,0.167,0.25$, and 0.5 moles of azacrown ether per mole of chitosan at equilibrium as a function of $\mathrm{pH}$ and $\mathrm{DAC}$ content.

\subsubsection{Swelling Kinetics}

The pH-dependent swelling kinetics was examined. It could be observed that the swelling of the Ch-DAC film hydrogels reaches maximum in about $100 \mathrm{~min}$ (Figure 7). The first three swelling measurements were made within this time, whereby the water solution had already diffused throughout the entire film strips, as shown by their soft, rubbery consistency [29]. Thus, the rate-determining process during the rest of the entire time that the swelling process was being monitored (about 7 days), was the stress relaxation of the chitosan film networks responding to the osmotic swelling pressure. The measurements that were made after a total of $360 \mathrm{~min}$, i.e., $12 \mathrm{~h}$ and beyond produced no significant weight changes. 


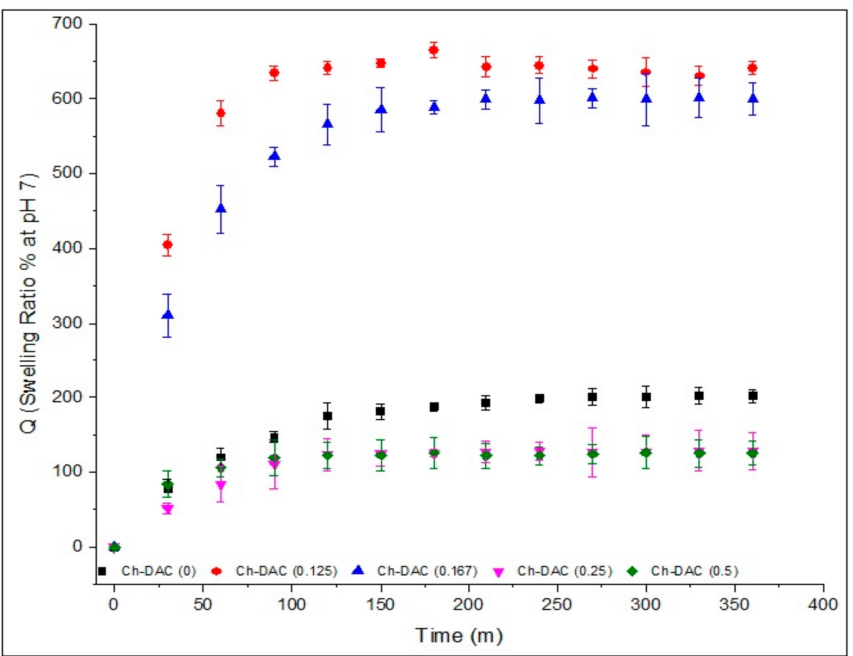

(a)

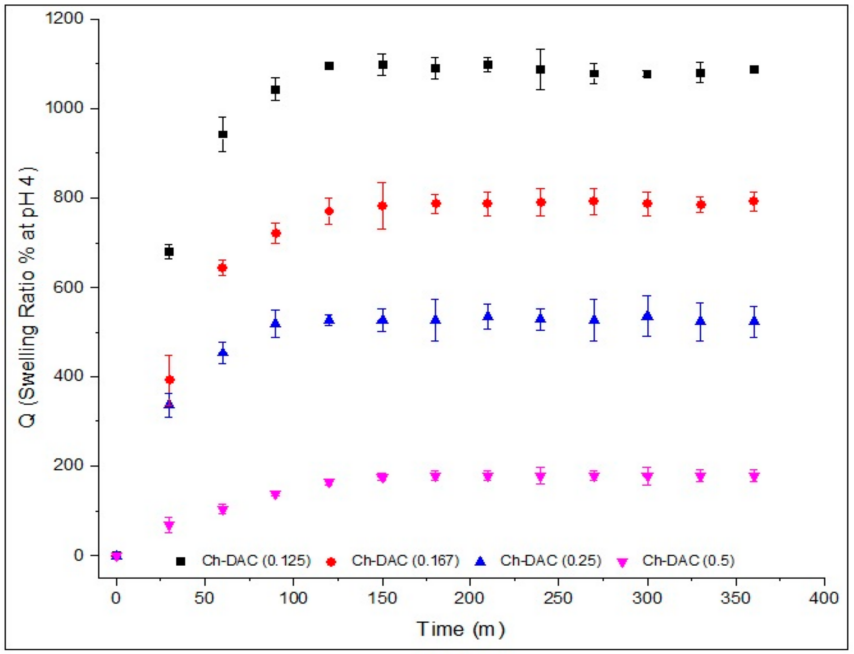

(b)

Figure 7. Swelling isotherms for Ch-DAC films (a) in water solution of $\mathrm{pH} 7$ and (b) in water solution of $\mathrm{pH} 4$.

To examine the swelling kinetics, Schott H. [39] proposed a model for second-order kinetics of swelling. Accordingly, it has been shown that the swelling rate of semi-crystalline or amorphous polymers such as gelatin and cellulose which is controlled by stress relaxation, follows second-order kinetics $[40,41]$. The same can be assumed to be applicable to chitosan-based polymer matrices because their swelling reaches a state of equilibrium [42,43]. Namely, as the network is swollen by solvent absorption, the chains between crosslinking points assume elongated conformations, and a force akin to the elastic retractive force in rubber consequently develops in the opposition to the swelling process [44]. As swelling proceeds, this force increases, and the diluting force decreases. A state of equilibrium swelling is reached in which these two forces are in balance. The useful equation that represents the data in linear form for the entire swelling period is given as [41]:

$$
\frac{t}{W}=A+B t
$$


At long swelling times, $B t \gg A$ and the slope $B=1 / W_{\max }$ is identified as the reciprocal of the maximum or equilibrium swelling [39]. This equation leads to the establishment of Equation (6) [43]:

$$
\frac{t}{W}=\frac{1}{k_{s} W_{\max }^{2}}+\frac{1}{W_{\max }} t,
$$

where $k_{s}$ stands for second-order swelling kinetics and is equal to $1 / A W_{\max }^{2}$, the units of $k$ are concentration $^{-1}$ time $^{-1}$, which are the correct dimensions for a second-order specific rate constant. $W_{\max }$ is the maximum swelling, and $W$ is the swelling at time $t$. To test this model, $t / W$ vs. $t$ graphs are plotted and the linear regressions of the lines of each swelling curve at all the $\mathrm{pHs}$ investigated indicate that they all obey the second order kinetics and $B t \gg A$.The fit was uniform and the linear correlation coefficients for all Ch-DAC films studied exceeded 0.99. Representative graphs are presented in the Supplementary Material Figures S3 and S4 from which it can be seen that $1 / A W_{\max }^{2}$ approaches zero indicating the short period with which equilibrium swelling is reached Figure S4). From the slope and intercepts of the lines, the maximum swelling, $W_{\max }$, and the swelling rate constant, $k_{s}$, are calculated and reported in Table S2. The table values show that the results of the kinetic model agree with swelling experiments. It is also interesting to observe in Figure 8 that with respect to $k_{s}$, for each $\mathrm{pH}$ value, the rate of swelling to reach EWC decreases with increasing DAC content. This could be due to increasing hydrophobicity of the film surfaces [45] because DAC was water insoluble and may be responsible for this observation. Likewise, higher crosslinking led to formation of a more rigid network with smaller pore volumes. However, going from $\mathrm{pH}$ value of 2 up to 10, the rate of swelling decreases significantly. This confirms that the $\mathrm{pH}$ of the adsorption media significantly affect the rate of swelling as was previously noted.

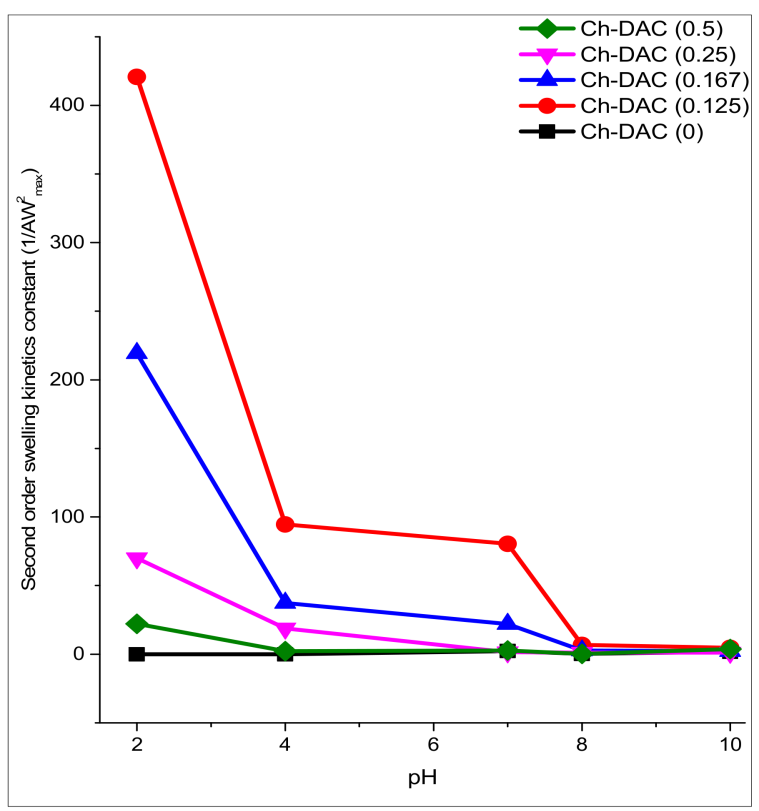

Figure 8. Second order swelling kinetics parameter. The graph shows that both DAC content and the solution $\mathrm{pH}$ influence the swelling behavior of the films.

\section{Discussion}

The crosslinked Ch-DAC films present a two-phase property behavior in properties in relation to the DAC molar ratio (Figure 9). At low DAC content $(<0.2 \mathrm{moles} / \mathrm{mole} \mathrm{Ch})$, the behavior is consistent with a highly branched chitosan gel with low crosslinking density (Figure 10a); at higher DAC molar ratio ( $>0.2 \mathrm{moles} / \mathrm{mole} \mathrm{Ch}$ ) the behavior suggests a highly crosslinked DAC- Ch topology (Figure 10b). The microstructural properties of the films, viz-a-viz specific surface area, total pore volume and 
micropore volume as a function of DAC content all indicate that the trend changes at a a threshold molar content of $[\mathrm{DAC}]<0.2$ moles/mole. Swelling capacity within the initial stage is high for all $\mathrm{pH}$ values tested. This behavior is consistent with slightly crosslinked topology and a predominance of grafts on the main chain (Figure 10a). This topology is also consistent with the internal plasticization effect of DAC revealed by the depression in this range of molar ratio.

Above the threshold molar content of 0.2 , â reverse trend is evident. Specific surface area, total pore volume, micropore volume, as well as glass transition temperature exhibit a decreasing trend with increasing DAC molar content. This trend is consistent with high crosslinking density and the fact that swelling capacity decreases significantly. The appearance of a second degradation temperature at higher DAC content may also suggest that at higher DAC content the materials might possess two distinguishable thermal phases.

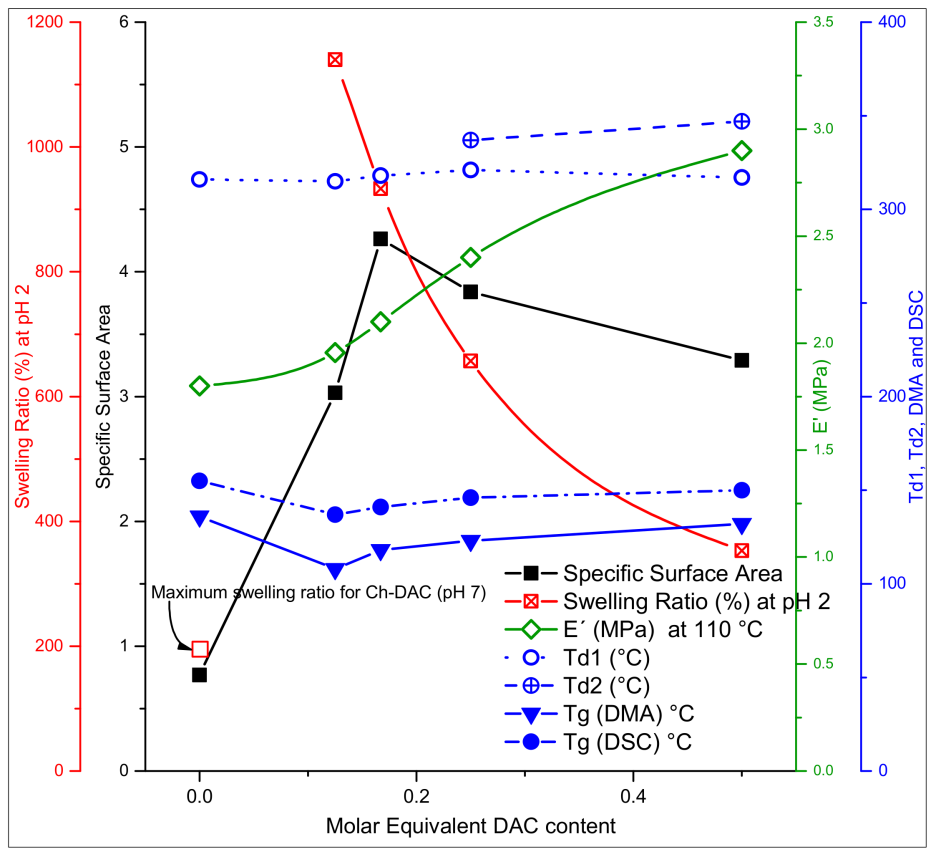

Figure 9. A summary diagram illustrating the interrelationship behaviors among various physical properties of the films.
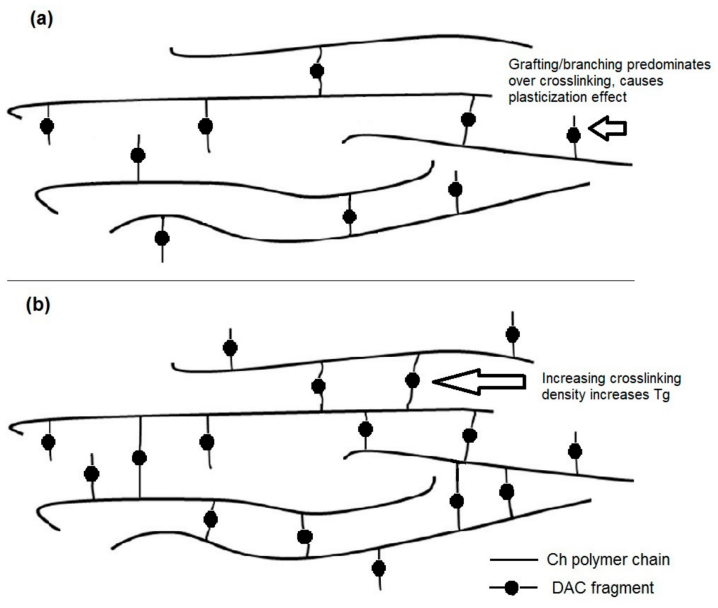

Figure 10. Model diagram showing (a) graft network polymer with light crosslinking and (b) highly crosslinked network polymer. 


\section{Conclusions}

The study showed that the microstructural morphology, thermal and swelling properties of chitosan are influenced by its crosslinking with azacrown ether. The evidence of two distinct trends in the thermal, structural and swelling properties of the crosslinked chitosan gel series with increasing DAC, suggests the predominance of two distinct network topologies around a threshold DAC content of 0.2 mole of DAC per mole of chitosan. It is proposed that below this threshold molar ratio, the chitosan networks are highly branched as revealed by the dominating plasticization effect. Above this threshold molar ratio, the $T_{g}$ rises systematically with DAC content revealing increased crosslinking density. The trends in specific surface area, swelling properties and kinetics and tensile storage modulus $\left(E^{\prime}\right)$ are consistent with this interpretation. Namely, initial incorporation of azacrown ether highly increases the network swelling capacity, but increasing the amount steadily lowers the swelling capacity. The ability to tailor specific surface area, swelling capacity and viscoelastic properties with DAC content and the resulting network topology is a promising step towards the molecular design of DAC-crosslinked chitosan gels for heavy metal adsorption. Metal adsorption studies with this series of Ch-DAC is the topic of a subsequent publication.

Supplementary Materials: The following are available online at www.mdpi.com/2305-7084/1/2/16/s1, Figure S1: Variation of water content (EWC \%) for Ch-DAC films reacted with $0,0.125,0.167,0.25$ and 0.5 moles of azacrown ether per mole of chitosan at equilibrium as a function of $\mathrm{pH}$ and DAC content, Figure S2: Swelling isotherms for Ch-DAC films reacted with 0, 0.125, 0.167, 0.25, and 0.5 moles of azacrown ether per mole of chitosan: (a) Equilibrium water content (\%); (b) to (f) is Swelling ratio (\%); Figure S3: Plots of $t / W$ vs. $t$ for swelling rates of chitosan and chitosan-DAC films as a function of $\mathrm{pH}$. Table S1: Maximum degradation temperature, DMA and DSC values for the Ch-DAC films as a function of reacting ratio, Table S2: Swelling parameters of chitosan and Ch-DAC chitosan film hydrogels at various $\mathrm{pH}$ values.

Acknowledgments: J. Toeri thanks the German Academic Exchange Program (DAAD) and the National Commission for Science, Technology, and Innovation of Kenya (NACOSTI) for the scholarship. J. Toeri also thanks Meru University of Science and Technology (MUST) for the leave of study. The authors thank Malte Buber of the University of Freiburg for acquiring the TGA data and Andreas Warmbold of the Freiburg Materials Center, University of Freiburg (FMF) for acquiring the BET data.

Author Contributions: Julius Toeri conceived the work; Julius Toeri and Marie-Pierre Laborie designed the experiments; Julius Toeri performed the experiments; Julius Toeri analyzed the data; Julius Toeri and Marie-Pierre Laborie interpreted the data and wrote the manuscript.

Conflicts of Interest: The authors declare no conflict of interest.

\section{References}

1. Alexandratos, S.D.; Stine, C.L. Synthesis of ion-selective polymer-supported crown ethers: A review. React. Funct. Polym. 2004, 60, 3-16. [CrossRef]

2. Wang, M.; Yan, F.; Fang, Z.; Zhang, Y. Preparation of chitosan-graft-benzo-15-crown-5 ether film for heavy metal ions separation. Desalin. Water Treat. 2017, 81, 143-151. [CrossRef]

3. Mututuvari, T.M.; Tran, C.D. Synergistic adsorption of heavy metal ions and organic pollutants by supramolecular polysaccharide composite materials from cellulose, chitosan and crown ether. J. Hazard. Mater. 2014, 264, 449-459. [CrossRef] [PubMed]

4. Al-Sarra, I.A.; Alanazi, F.K.; Radwan, A.A. Chitosan Derivative, A Method for Its Preparation and Its Use as An Adsorption Agent. WIPO Patent WO2013000567, 4 January 2013.

5. Malkondu, S.; Kocak, A.; Yilmaz, M. Immobilization of Two Azacrown Ethers on Chitosan: Evaluation of Selective Extraction Ability Toward Cu(II) and Ni(II). J. Macromol. Sci. Part A 2009, 46, 745-750. [CrossRef]

6. Yang, Z.; Liu, L.; Zhang, L.; Wang, Y. Preparation and adsorption behavior of macrocyclic tetra-amine derivatives of chitosan. J. Appl. Polym. Sci. 2005, 98, 407-412. [CrossRef]

7. Zhang, S.-Q.; Wang, Y.-T.; Tang, Y.-R. Studies of some ultratrace elements in antarctic water via crown ether crosslinked chitosan. J. Appl. Polym. Sci. 2003, 90, 806-809. [CrossRef]

8. Changhong, P.; Weijun, Y.; Motang, T. Chemical modification of chitosan: Synthesis and characterization of chitosan-crown ethers. J. Appl. Polym. Sci. 2003, 87, 2221-2225. [CrossRef] 
9. Peng, C.; Wang, Y.; Tang, Y. Synthesis of crosslinked chitosan-crown ethers and evaluation of these products as adsorbents for metal ions. J. Appl. Polym. Sci. 1998, 70, 501-506. [CrossRef]

10. Peng, C.; Wang, Y.; Tan, S.; Cheng, G. Preparation of Chitosan Derivatives. Synthesis of N-Schiff Base Type and N-Secondary Amino Type Chitosan-Crown Ethers. Polym. J. 1998, 30, 843-845. [CrossRef]

11. Tang, X.-H.; Tan, S.-Y.; Wang, Y.-T. Study of the synthesis of chitosan derivatives containing benzo-21-crown-7 and their adsorption properties for metal ions. J. Appl. Polym. Sci. 2002, 83, 1886-1891. [CrossRef]

12. Yang, Z.; Cheng, S. Synthesis and characterization of macrocyclic polyamine derivative of chitosan. J. Appl. Polym. Sci. 2003, 89, 924-929. [CrossRef]

13. Yang, Z.; Shu, J.; Zhang, L.; Wang, Y. Preparation and adsorption behavior for metal ions of cyclic polyamine derivative of chitosan. J. Appl. Polym. Sci. 2006, 100, 3018-3023. [CrossRef]

14. Yang, Z.; Wang, Y.; Tang, Y. Preparation and adsorption properties of metal ions of crosslinked chitosan azacrown ethers. J. Appl. Polym. Sci. 1999, 74, 3053-3058. [CrossRef]

15. Yang, Z.; Wang, Y.; Tang, Y. Synthesis and adsorption properties for metal ions of mesocyclic diamine-grafted chitosan-crown ether. J. Appl. Polym. Sci. 2000, 75, 1255-1260. [CrossRef]

16. Yang, Z.; Yuan, Y.; Wang, Y. Synthesis and evaluation of chitosan aryl azacrown ethers as adsorbents for metal ions. J. Appl. Polym. Sci. 2000, 77, 3093-3098. [CrossRef]

17. Yang, Z.; Zhuang, L.; Tan, G. Preparation and adsorption behavior for metal of chitosan crosslinked by dihydroxy azacrown ether. J. Appl. Polym. Sci. 2002, 85, 530-535. [CrossRef]

18. Dhakal, R.P.; Oshima, T.; Baba, Y. Synthesis of Unconventional Materials Using Chitosan and Crown Ether for Selective Removal of Precious Metal Ions. World Acad. Sci. Eng. Technol. 2009, 56, 204-208.

19. Zhang, X.; Ding, S.; Wang, Y.; Feng, X.; Peng, Q.; Ma, S. Synthesis and adsorption properties of metal ions of novel azacrown ether crosslinked chitosan. J. Appl. Polym. Sci. 2006, 100, 2705-2709. [CrossRef]

20. Yi, Y.; Wang, Y.; Liu, H. Preparation of new crosslinked chitosan with crown ether and their adsorption for silver ion for antibacterial activities. Carbohydr. Polym. 2003, 53, 425-430. [CrossRef]

21. Radwan, A.A.; Alanazi, F.K.; Alsarra, I.A. Microwave Irradiation-Assisted Synthesis of a Novel Crown Ether Crosslinked Chitosan as a Chelating Agent for Heavy Metal Ions (M+n). Molecules 2010, 15, 6257-6268. [CrossRef] [PubMed]

22. Tan, S.; Wang, Y.; Peng, C.; Tang, Y. Synthesis and adsorption properties for metal ions of crosslinked chitosan acetate crown ethers. J. Appl. Polym. Sci. 1999, 71, 2069-2074. [CrossRef]

23. Wan, L.; Wang, Y.; Qian, S. Study on the adsorption properties of novel crown ether crosslinked chitosan for metal ions. J. Appl. Polym. Sci. 2002, 84, 29-34. [CrossRef]

24. Zhikuan Yang, Y.Y. Synthesis, characterization, and adsorption properties of chitosan azacrown ethers bearing hydroxyl group. J. Appl. Polym. Sci. 2001, 81, 1793-1798. [CrossRef]

25. Ding, S.; Zhang, X.; Feng, X.; Wang, Y.; Ma, S.; Peng, Q.; Zhang, W. Synthesis of N,N'-diallyl dibenzo 18-crown-6 crown ether crosslinked chitosan and their adsorption properties for metal ions. React. Funct. Polym. 2006, 66, 357-363. [CrossRef]

26. Bradshaw, J.S.; Krakowiak, K.E.; Izatt, R.M. Aza-Crown Macrocycles; Wiley: New York, NY, USA, 1993.

27. Krakowiak, K.E.; Bradshaw, J.S.; Zamecka-Krakowiak, D.J. Synthesis of aza-crown ethers. Chem. Rev. 1989, 89, 929-972. [CrossRef]

28. Izatt, R.M.; Pawlak, K.; Bradshaw, J.S.; Bruening, R.L. Thermodynamic and Kinetic Data for Macrocycle Interaction with Cations, Anions, and Neutral Molecules. Chem. Rev. 1995, 95, 2529-2586. [CrossRef]

29. Toeri, J.; Osorio-Madrazo, A.; Laborie, M.-P. Preparation and Chemical/Microstructural Characterization of Azacrown Ether-Crosslinked Chitosan Films. Materials 2017, 10, 400. [CrossRef] [PubMed]

30. Flory, P.J. Statistical Mechanics of Swelling of Network Structures. J. Chem. Phys. 1950, 18, 108-111. [CrossRef]

31. Rohindra, D.R.; Nand, A.V.; Khurma, J.R. Swelling properties of chitosan hydrogels. S. Pac. J. Nat. Appl. Sci. 2004, 22, 32-35. [CrossRef]

32. Toeri, J.; Laborie, M.-P. Synthesis and Characterization of Macrocyclic Polyether N,N'-Diallyl-7,16diaza-1,4,10,13-tetraoxa-dibenzo-18-crown-6. Molecules 2016, 21, 171. [CrossRef] [PubMed]

33. Dubinin, A.M.M. A study of the porous structure of active carbons using a variety of methods. Q. Rev. Chem. Soc. 1955, 9, 101-114. [CrossRef]

34. Ahn, J.-S.; Choi, H.-K.; Cho, C.-S. A novel mucoadhesive polymer prepared by template polymerization of acrylic acid in the presence of chitosan. Biomaterials 2001, 22, 923-928. [CrossRef] 
35. Dong, Y.; Ruan, Y.; Wang, H.; Zhao, Y.; Bi, D. Studies on glass transition temperature of chitosan with four techniques. J. Appl. Polym. Sci. 2004, 93, 1553-1558. [CrossRef]

36. Butler, M.F.; Clark, A.H.; Adams, S. Swelling and Mechanical Properties of Biopolymer Hydrogels Containing Chitosan and Bovine Serum Albumin. Biomacromolecules 2006, 7, 2961-2970. [CrossRef] [PubMed]

37. Tasselli, F.; Mirmohseni, A.; Seyed Dorraji, M.S.; Figoli, A. Mechanical, swelling and adsorptive properties of dry-wet spun chitosan hollow fibers crosslinked with glutaraldehyde. React. Funct. Polym. 2013, 73, 218-223. [CrossRef]

38. Somasundaran, P. (Ed.) Encyclopedia of Surface and Colloid Science; CRC Press: Boca Raton, FL, USA, 2006.

39. Schott, H. Swelling kinetics of polymers. J. Macromol. Sci. Part B 1992, 31, 1-9. [CrossRef]

40. Ofner, C.M.; Schott, H. Swelling Studies of Gelatin I: Gelatin Without Additives. J. Pharm. Sci. 1986, 75, 790-796. [CrossRef] [PubMed]

41. Ofner, C.M.; Schott, H. Swelling Studies of Gelatin II: Effect of Additives. J. Pharm. Sci. 1987, 76, 715-723. [CrossRef] [PubMed]

42. Branca, C.; Auditore, L.; Loria, D.; Trimarchi, M.; Wanderlingh, U. Radiation synthesis and characterization of poly(ethylene oxide)/chitosan hydrogels. J. Appl. Polym. Sci. 2013, 127, 217-223. [CrossRef]

43. Katime, I.; Valderruten, N.; Quintana, J.R. Controlled release of aminophylline from poly (N-isopropylacrylamideco-itaconic acid) hydrogels. Polym. Int. 2001, 50, 869-879. [CrossRef]

44. Flory, P.J. Principles of Polymer Chemistry, 1st ed.; Cornell University Press: Ithaca, NY, USA, 1953.

45. Guibal, E. Interactions of metal ions with chitosan-based sorbents: A review. Sep. Purif. Technol. 2004, 38, 43-74. [CrossRef]

(C) 2017 by the authors. Licensee MDPI, Basel, Switzerland. This article is an open access article distributed under the terms and conditions of the Creative Commons Attribution (CC BY) license (http:/ / creativecommons.org/licenses/by/4.0/). 